\title{
Physiological, Biochemical and Modern Biotechnological Approach to Improvement of Rauwolfia serpentina
}

\author{
Poonam , Shipra Agrawal and Sanjay Mishra* \\ School of Biotechnology, IFTM University, Lodhipur Rajput, Delhi Road (NH-24), Moradabad 244102, U.P., \\ India
}

\begin{abstract}
Rauwolfia serpentina also known as Sarpagandha (Apocynaceae) is an integral part of Ayurvedic medical system in India for over centuries for the treatment of various ailments. The leaves and roots ofRauwolfiaserpentina contain alkaloids which are secondary metabolites. Major alkaloids identified are Reserpine, Rauwolfine, Serpentine, Sarpagine, Ajmaline, Yohimbine and Ajmalicine.The present paper is an overview of the studies concerning with physiological, biochemical and modern biotechnological approach to improvement of Rauwolfiaserpentina.
\end{abstract}

Keywords: Rauwolfia serpentina; medicinal plant; micro-propagation; tissue culture; Leaf / Root explants; Growth regulators; Auxins; Reserpine.

\section{Introduction}

Rauwolfia serpentina is an important medicinal plant (shrub) belonging to the Apocynaceae family. This plant is occurring naturallyin India and Bangladesh and is found to grow wild in the Asian continent. It has been reported to contain 50 indole alkaloids that are mainly localized in the root bark[1].Among these alkaloids, reserpine, yohimbine, serpentine, deserpidine, ajmalicine and ajmaline are used to treat hypertension [2] and breast cancers[3].Reserpine, used as a natural tranquilizer was found to have several times greater hypertensive activity than the crude plant extract [4]. Rauwolfia serpentina holds an important position in the pharmaceutical world because of its immense anti-hypertensive properties resulting from the presence of reserpine in the oleoresin fraction of the roots. Poor seed viability, low seed germination rate, and enormous genetic variability are the major constraints for the commercial cultivation of $R$. serpentine through conventional mode. Many higher plants are major sources of natural products used as pharmaceuticals, agrochemicals, flavour and fragrance ingredients, food additives, and pesticides [5]. The search for new plant derived chemicals should thus be a priority in current and future efforts toward sustainable conservation and rational utilization of biodiversity [6]. In the search for alternatives to production of desirable medicine compounds from plants, biotechnological approaches, specifically, plant tissue cultures, are found to have potential as a supplement to traditional agriculture in the industrial production of bioactive plant metabolites [7]. Cell suspension culture systems could be used for large scale culturing of plant cells from which secondary metabolites could be extracted. The advantage of this method is that it can ultimately provide a continuous, reliable source of natural products. The roots, the leaves and the juice of R.serpentina have been considered of medicinal importance from the very early times and have attracted the attention of the practitioners of the indigenous system of medicine. It has been used as an anthelmintic, as an antidote against snake bite and bites of other poisonous insects, in diarrhoea, dysentery, cholera and also as an ecbolic.

Rauwolfia root is reported to contain $0.7-3.0 \%$ of total alkaloids in the dry mass and the amount vary with time and source of collection. The rate of plant propagation is important for commercial cultivation to meet the pharmaceutical demand for reserpine. Chemical synthesis of reserpine has not been adopted due to its high cost compared to extraction from the natural source [8]. While roots of R.serpentinais the main source of the alkaloids mentioned above, indiscriminate harvesting of the roots has threatened the survival of the plant. However, high demand for the alkaloids necessitates rapid production of roots within a short time frame. Therefore, root cultures are a potentially useful in vitro system for commercial production of secondary metabolites.

Rauwolfia serpentine roots are generally obtained through shoot organogenesis[9]callus morphogenesis, [10] or by Agrobacterium rhizogenes-mediated transformation [11].

The induction of callus growth and subsequent differentiation and organogenesis is accomplished by the differential application of growth regulators and the control of conditions in the culture medium. With the stimulus of endogenous growth substances or by addition of exogenous growth regulators to the nutrient medium, cell division, cell growth and tissue differentiation are induced. There are many reports on the regeneration of various medicinal plants via callus culture. Satheesh and Bhavanandan [12] have reported the regeneration of shoots from callus of Plumbagorosea using appropriate concentrations of auxins and cytokinins. Mantell and Hugo [13] have also reported a high frequency of shoot, root, and micro tuber production from 
Dioscoreaalatadepending on the culture medium used, the type of explant from which the calli originated, and the photoperiod.

\section{Regeneration through somatic embryogenesis:}

Somatic embryogenesis is a process where groups of somatic cells/tissues lead to the formation of somatic embryos which resemble to the zygotic embryos of intact seeds and can grow into seedlings on suitable medium. Plant regeneration via somatic embryogenesis from single cells, that can be induced to produce an embryo and then a complete plant, has been demonstrated in many medicinal plant species. Ghosh and Sen[14] reported regeneration and somatic embryogenesis in Asparagus cooperion MS medium.

\section{Genetic Transformation:}

The recent advances and developments in plant genetics and recombinant DNA technology have helped to improve and boost research into secondary metabolite biosynthesis. A major line of research has been to identify enzymes of a metabolic pathway and then manipulate these enzymes to provide better control of that pathway. Transformation is currently used for genetic manipulation of more than 120 species of at least 35 families, including the major economic crops, vegetables, ornamental, medicinal, fruit, tree and pasture plants, using Agrobacteriummediated or direct transformation method [15]. However, Agrobacterium mediated transformation offers several advantages over direct gene transfer methodologies (particle bombardment, electroporation, etc), such as the possibility to transfer only one or few copies of DNA fragments carrying the genes of interest at higher efficiencies with lower cost and the transfer of very large DNA fragments with minimal rearrangement[16].

The gram-negative soil bacteria, Agrobacterium tumefaciens, and the related species, A. rhizogenes, are causal agents of the plant diseases crown gall tumour and hairy root, respectively Agrobacterium rhizogenes has been used regularly for gene transfer in many dicotyledonous plants[17]. Plant infection with this bacterium induces the formation of proliferative multibranched adventitious roots at the site of infection; the so-called 'hairy roots' [18].This infection is followed by the transfer of a portion of DNA i.e. T-DNA, known as the root inducing plasmid (Riplasmid), to the plant cell chromosomal DNA. The research is going for the application of plant transformation and genetic modification using A. rhizogenes, in order to boost production of those secondary metabolites, which are naturally synthesized in the roots of the mother plant. Transformed hairy roots mimic the biochemical machinery present and active in the normal roots, and in many instances transformed hairy roots display higher product yields.

So far, no protocol has been published for in vitro growth of R.serpentinaroots directly from leaf explants using growth regulators. The present study was undertaken to develop a protocol for rapid induction of $R$. serpentinaroots using the leaf of the plant as the starting material. Genetic transformation would be a powerful tool for enhancing the productivity of novel secondary metabolites of limited yield. Hairy roots, transformed with Agrobacteriumrhizogenes, have been found to be suitable for the production of secondary metabolites because of their stable and high productivity in hormone-free culture conditions. A number of plant species including many medicinal plants have been successfully transformed with Agrobacterium rhizogenes.

\section{Conservation of endangered medicinal plant using Agrobacterium species:}

Different techniques adopted so far to conserve this endangered medicinal plant, Agrobacterium rhizogenes-mediated 'hairy root' cultures of different Rauwolfia species have attracted the major attention. These genetically transformed 'hairy root' cultures have already provided much evidence of their suitability as an alternative production source, and offer the advantages of genetic stability and consistency in the product yield over a longer period of time, faster growth rate, autonomy for auxin biosynthesis and minimal nutritional requirements, leading to cost reduction and the potential to synthesize secondary metabolites similar to those of the parent plants [19]. Transgenic hairy root cultures have revolutionized the role of plant tissue culture in secondary metabolite production. They are unique in their genetic and biosynthetic stability, faster in growth, and more easily maintained. Using this methodology a wide range of chemical compounds has been synthesized $[20,21]$. Advances in tissue culture, combined with improvement in genetic engineering, specifically transformation technology, have opened new avenues for high volume production of pharmaceuticals, nutraceuticals, and other beneficial substances [22]. Recent advances in the molecular biology, enzymology, and fermentation technology of plant cell cultures suggest that these systems will become a viable source of important secondary metabolites. Genome manipulation is resulting in relatively large amounts of desired compounds produced by plants infected with an engineered virus, whereas transgenic plants can maintainconstant levels of production of proteins without additional intervention [23]. 


\section{Production of secondary metabolites:}

Plants are the traditional source of many chemicals used as pharmaceuticals. Most valuable phytochemicals are products of plant secondary metabolism. The production of secondary metabolites in vitro can be possible through plant cell culture [24].Successful establishment of cell lines have several advantages for mass cultivation of plant cells, major of those are as follows:

1. It gives better control for scale up of cell suspension cultures under defined parameters for the production of bioactive compounds.

2. Constant regulation of conditions at various stages of bioreactor operation is possible.

3. Handling of culture such as inoculation or harvest is easy and saves time.

4. Nutrient uptake is enhanced by submerged culture conditions which stimulate multiplication rate and higher yield of bioactive compounds and

5. Large number of plantlets is easily produced and can be scaled up.

Since the biosynthetic efficiency of populations varies, a high yielding variety should be selected as a starting material. The fundamental requirement in all this is a good yield of the compound, and reduced cost compared to the natural synthesis by the plants.

\section{Alkaloids:}

Alkaloids are complex organic molecules containing a heterocyclic nitrogen ring, which have been widely exploited for their diverse pharmacological properties. Such compounds are produced by many different organisms, including animals and microbes, but a particularly diverse array of alkaloids is produced by plants. Alkaloids are often isolated from plants and used as pure compounds, although they may also be administered as crude extracts. Approximately $10 \%$ of all plant species are thought to produce alkaloids as secondary metabolites, where they function predominantly in defenses against herbivores and pathogens. Although such ecochemical functions are important in their own right, most of the interest in alkaloids stems from their potent and specific pharmacological effects in humans, and this is also the reason why metabolic engineering of alkaloid biosynthesis is an important area of research.

\section{Quantitative and Qualitative analysis of indolealkaloids:}

One of the most effective methods for the qualitative and quantitative analysis of alkaloids is HPLC because of its high resolution power and automatisation. Different stationary phases were used in the past for normal phase packing, e.g. aluminum oxide [25] and silica gel [26], or reversed-phase materials like RP8[27] or RP18 [28,29].

In some cases ion-exchange columns were used to determine alkaloids such as the highly polar polyhydroxy alkaloids or opium alkaloids. The use of acetonitrile-water mixtures with ammonium formiate as eluent allowed the coupling to MS. When diluted hydrochloric acid use as an eluent for ion-exchange chromatography, the direct measurement of biological activity could be performed after addition of phosphate to the obtained fractions [30]. Liquid chromatography on reversed-phase columns is nowadays "state of the art" in the analysis of natural products and especially alkaloids. The most attractive advantage of RP chromatography is deproto the ability to determine simultaneously a wide variety of compounds which differ markedly in men molecular structure, molecular mass, polarity and acidity/basicity. Only very polar compounds, like the above mentioned polyhydroxy alkaloids or derivatives lacking a UV absorbing chromophore are difficult to separate with reversed phase columns followed by conventional UV detection. To overcome these problems the application of gas chromatography is an excellent alternative [31]. However for HPLC-analysis of basic alkaloids with reversed-phase material no generally applicable method exists. As RP18 material provides the highest affinity to lipophilic structures, such as the carbon skeletons of monoterpenoidindole alkaloids, and also shows the best shielding of the silica gel [32,33], we decided to apply an RP18 column for the optimization of the buffer system which we had developed for a more alter a general application to alkaloid analysis. Unless otherwise stated, for all initial experiments we used isocratic conditions with acetonitrile and a $25 \mathrm{mM}$ phosphate buffer as eluents. The formation of uncharged and thus more lipophilic ion pairs depends on:

(i) The concentration of the counter-ions,

(ii) The $\mathrm{pH}$ value of the buffer,

(iii) The dissociation constant of the ion pair reagents and analytes, and

(iv) The dielectric constant of the eluent mixture which favors the ionic mechanisms between the cationic alkaloids and the anionic sulfonic acid [34].The lower the dielectric constants of the solvents used, the higher are the formation rates of ion pairs. For this reason we chose acetonitrile as eluent and an acidic buffer solution which provided a strong dissociation of the sulfonic acid and a quantitative protonation of the basic alkaloids. 


\section{Conservation through cryopreservation:}

The cryopreservation of in-vitro cultures of medicinal plants is a useful technique. Cryopreservation is long-term conservation method in liquid nitrogen $\left(-196{ }^{\circ} \mathrm{C}\right)$ in which cell division and metabolic and biochemical processes are arrested. A large number of cultured materials can be stored in liquid nitrogen. Since whole plants can regenerate from frozen culture, cryopreservation provides an opportunity for conservation of endangered medicinal plants. For example, low temperature storage has been reported to be effective for cell cultures of medicinal and alkaloid producing plants such as Rauvollfia serpentina, D. lanalta, and A. belladonna and Hyoscyamus spp. When plants are regenerated and no abnormality is seen either in fertility or in alkaloid content, the materials can be stored using cryopreservation methods. Cryopreservation has been used successfully to store a range of tissue types, including meristems, anthers/pollens, embryos, calli and even protoplasts. However, the system absolutelyl depends on the availability of liquid nitrogen methods.

There recently optimized protocol offers an impeccable end to end method from the establishment of aseptic cultures to in-vitro plantlet production employing semisolid as well liquid nutrient culture medium and assessment of their genetic fidelity using polymerase chain reaction based rapid amplification of polymorphic DNA analysis. In vitro shoots multiplied on Murashigeand Skoog basal liquid nutrient medium and extraction of indole alkaloid particularly reserpine, ajmaline and ajmalicine and their simultaneous quantitation performe using monolithic reverse phase high-performance liquid chromatography (HPLC) [35]. Numerous factors are reported to influence the success of in-vitro propagation of different medicinal plants [36]. The effects of auxins and cytokinins on shoot multiplication of various medicinal plants have been reported[37], has shown that 6Benzylaminopurine (BA), stimulates the development of the axillary meristems and shoot tips of Atropabelladonna[37,38] observed a rapid proliferation rate in Picrorhizakurroausing kinetin . Direct plantlet regeneration from male inflorescences of medicinal yam on medium supplemented with kinetin has also be reportedby[39].The highest shoot multiplication of Nothapodytesfoetida is achieved on medium containing thidiazuron (TDZ)[40].Similarly, it has been observed that cytokinin is required, in optimal quantity, for shoot proliferation in many genotypes but inclusion of low concentration of auxins along with cytokinin triggers the rate of shoot proliferation [41].

Rauwolfia serpentine was reported to contain a large number of therapeutically useful indole alkaloids and these alkaloids are largely located in the roots. Hence, root biomass production of this plant could be of economic importance. Chemically, the root contains a number of alkaloids. The Quantitative determination of total alkaloids in this plant in tissue culture was reported by Shimolinain 1984. Mechanism for irradiance skeleton formation in the biosynthesis of secologanin and indole alkaloid in suspension culture of R. serpentine was well reported Uesatoin 1986. Quantitative estimation of Rutin in this plant was reported by Bharadwajin 1988. Expression of enzymatically active cloned strictosidine synthesis from R. serpentina in Escherichia coli was reported by Kutchan in 1989. Biotransformation of ajmaline in plant cell culture and new indole alkaloids raumacline and $\mathrm{N}(\beta)$-methyl raumaclin were reported [42] reflecting high yield formation of arbutin from Hydroquinone by cell suspension culture. The quality and quantity of auxins supplemented to the culture of leaf explants affected the type of regeneration as well as secondary metabolite production, as has been reported in several other organ cultures [43]. The low reserpine content of the in vitro regenerated roots found in this work may be attributed to two factors. First, roots regenerated from leaf explants which are known for low alkaloid content. Second, the regenerated roots lacked a bark and the bark of the plant is reported to contain higher alkaloid content than the root. Chemical synthesis of reserpine has not been adopted due to its high cost compared to extraction from the natural source [44] while roots of $R$. serpentine is the main source of the alkaloids mentioned above, indiscriminate harvesting of the roots has threatened the survival of the plant. However, high demand for the alkaloids necessitates rapid production of roots within a short time-frame. Therefore, root cultures are a potentially useful in vitro system for commercial production of secondary metabolites. Direct root induction from leaf explant as a method for the rapid root regeneration has been reported for several plants, viz, Lycopersiconesculentum [45], Nicotianatabacum[46]and Begonia[47].

\section{Effect of R. Serpentina on differentmedium:}

R. serpentinaexplants incubated under dark conditions produced thin roots which indicated that photoperiod also influences invitro root growth. Overall, therefore, the results show that solid MS media is good for root induction from the leaf explants of $R$. serpentina. On the other hand, liquid MS media inhibited percentage of regeneration, root growth, root length, and reserpine content. This effect may due to the production of some metabolites that accumulated in the liquid culture and exerted inhibitory effect during the course of root growth. However, this hypothesis will need to be verified in future studies.

The cell suspensions culture of Rauwolfiaserpentina(L.) Kurzhas been established since 1980, and the culture is presently optimized as one of the most efficient and best characterized systems when the phytochemistry and enzymology of indole alkaloids, the biotransformation capabilities, and the application for hybrid generation is concerned [48].A series of enzymes were purified and characterized, which exhibit 
different functions in alkaloid biosynthesis and biotransformation [49, 50, 51]. All these information prompted us to utilize cell suspension to biotransform Paclitaxel, which might lead to polar hydroxylated or glycosidated Paclitaxel derivatives.

Large-scale plant tissue culture is found to be an attractive alternative approach to traditional methods of plantation as it offers a controlled supply of biochemical independent of plant availability[52, 53] detailed the impact of specific engineering-related factors on cell suspension cultures. Current developments in tissue culture technology indicate that transcription factors are efficient new molecular tools for plant metabolic engineering to increase the production of valuable compounds [54]. In vitro cell culture offers an intrinsic advantage for foreign protein synthesis in certain situations since they can be designed to produce therapeutic proteins, including monoclonal antibodies, antigenic proteins that act as immunogenes, human serum albumin, interferon, immuno-contraceptive protein, ribosome unactivatortrichosantin, antihypersensitive drug angiotensin, leuenkephalin neuropeptide, and human haemoglobin [55].

\section{R. serpentina (as a therapeutic agent):}

$R$. serpentina is therapeutically used as a sedative, a hypnotic drug and in hypertension. About $0.1 \%$ of the active principle reserpine which is an indole alkaloid is present in the root [56]. Homoeopathy is a holistic system of therapy which works at reinforcing the body's own natural capacity to heal and achieve a gentle and lasting cure. Mother tinctures (MQ) are defined as the original tincture prepared with the aid of alcohol, directly from the crude drug. They are the precursors of the corresponding potencies of the respective drug and the starting point for the production of most homoeopathic medicines [57].Alkaloids are very important in medicine and constitute most of the valuable drugs [58].

R. serpentina is said to have the following pharmacological attributes. (1) By action on the vaso motor centre, it leads to generalized vasodilatation, with a lowering of blood pressure. (2) By depressant action on the cerebral centres, it soothes the general nervous system. (3) It exerts a sedative action on the gastric mucosa and a stimulating action on the plain musculature of the intestinal tract. (4) It stimulates the bronchial musculature. A vague reference to the use of a tincture or alcoholic extract of the root of R. Serpentina, in cases of high blood pressure, was made in 1942 by Paranjpe. He claimed improvement, without any statistical backing, in most cases of hypertension; the hypotensive action was said to be particularly gratifying in elderly subjects- and in the case of the diastolic pressure; the tincture was said to be a good cough-sedative and diuretic. In two cases reported by Paranjpe, there had been a permanent reduction of blood pressure for well over a year, on occasional doses of the tincture.

\section{References}

[1] Klyushnichenko VE, Yakimov SA, Tuzova TP, Syagailo YV, Kuzovkina IN,WulfsonAN andMiroshnikov AI. Determination of indole alkaloids from $R$. serpentinaand $R$. vomitoriaby highperformance liquid chromatography and high-performance thinlayer chromatography.J of ChromatogrA. 1995; 704: 357-362.

[2] Von Poser G, Andrade HH, Da Silva KV, Henriques AT, Henriques JA. genotoxic, mutagenic and recombinogenic effects of Rauwolfia alkaloids. MutatRes J. 1990; 232: 37-43.

[3] Stanford JL, Martin EJ, Brinton LA, Hoover RN.Rauwolfia use and breast cancer: Acasecontrol study. J Natl Cancer Inst 1986; 76: 817-822.

[4] Pullaiah J. Medicinal plants in India, Vol. II. New Delhi: Regency Publ; 2002; pp 441-443.

[5] Balandrin, M.J. and J.A. Klocke, 1988. Medicinal, aromatic and industrial materials from plants.In Y.P.S. Bajaj (ed.), Biotechnology in Agriculture and Forestry. Medicinal and Aromatic Plant, vol. 4.Springer-Verlag, Berlin, Heidelberg, pp. 1-36.

[6] Phillipson, J.D. 1990. Plants as source of valuable products.In B.V. Charlwood, and M.J.C. Rhodes (eds.), Secondary Productsfrom Plant Tissue Culture. Oxford: Clarendon Press, pp. 1-21.

[7] RamachandraRao, S. and G.A. Ravishankar. 2002. Plant cell cultures: Chemical factories of secondary metabolites. Biotechnol. Adv. 20: 101-153.

[8] Farooqi AA, Sreeramu BS. Cultivation of medicinal and aromatic crops. Hyderabad: Uni Press; 2001; pp 210-211.

[9] Roja G, Heble MR. Indole alkaloids in clonal propagules ofRauwolfiaserpentina. Plant Cell Tissue Org Cult 1996; 44(2): 111 - 115.

[10] Pandey VP, Kudakasseril J, Cherian E, Patani G. Comparison of two methods for in vitro propagationofRauwolfia serpentine from nodal explants. Ind Drugs 2007; 44: 514-519.

[11] Banerjee S, Kukreja AK, Kahol AP, Kumar S.Bioreactor for enhancing the biomass of plant organs. 2003; US Pat 6589780B2.

[12] Satheesh Kumar K, Bhavanandan KV. Micropropagation of Plumbagorosea Linn.Plant Cell Tissue Organ Cult.1988; 15 : 275 - 8.

[13] Mantell SH, Hugo SA. Effects of photoperiod, mineral medium strength, inorganic ammonium, sucrose and cytokinin on root,shoot and microtuber development in shoot cultures of DioscoreaalataL. and D.bulbiferaL. yams. Plant Cell Tissue Organ Cult.1989; 16: 23-37.

[14] Ghosh BE, Sen S. Plant regeneration through somatic embryogenesis from spear callus culture of Asparagus cooperi Baker. Plant Cell Rep. 1991; 9: 667- 70.

[15] Birch RG. Plant transformation: Problems and strategies for practical application. Ann Rev Plant Physiol Plant Mol Biol. 1997; 48: 297- 326.

[16] Gheysen G, Angenon G, Montague MV. Agrobacterium-mediated plant transformation: a scientifically intriguing story with significant application. In: Lindsey K (ed). Transgenic Plant Research. Netherlands: Harwood Academic Press, 1998, pp1-33.

[17] Tepfer D. Genetic transformation using Agrobacterium rhizogenes.Physiol Plant. 1990; 79: 140-6.

[18] Chilton MD, Tepfer D, Petit A, David C, Casse- Delbart FT. Agrobacterium rhizogenes inserts T-DNA into the genome of the host plant root cells. Nature.1982; 295: 432-4. 
[19] Guillon S, Tremouillaux-Guiller J, Pati PK, Rideau M and Gantet P. Harnessing the potential of hairy roots: dawn of a new era. Trendsin Biotechnology 2006; 24: 403-409.

[20] Shanks, J.V. and J. Morgan. 1999. Plant hairy root culture. Curr.Opin.Biotechnol.10: 151-155.

[21] Giri, A. and M.L. Narasu. 2000. Transgenic hairy roots: recent trends and applications. Biotechnol. Adv. 18: 1-22.

[22] Hansen, G. and M.S. Wright. 1999. Recent advances in the transformation of plants. Trends Plant Sci. 4: $226-231$.

[23] Sajc, L., D. Grubisic, and G. Vunjak-Novakovic. 2000. Bioreactors for plant engineering: an outlook for further research. Biochem. Eng. J. 4: 89-99.

[24] Barz W, Ellis B. Potential of plant cell cultures for pharmaceutical production. In: Beal JL, Reinhard E (eds.) Natural Products asMedicinal Agents.Stuttgart, Hippokrates, 1981, pp 471

[25] G.D. Manners, J.A. Pfister, Phytochem. Anal.4 (1993) 14

[26] M. Huntin, A. Cave, J.P. Foucher, J. Chromatogr. 268 (1983) 125.

[27] Falkenhagen H, I.N. Kuzovkina, I.E. Alterman, L.A. Nikolaeva, J. Sto“ckigt, Nat. Prod.Lett.3 (1993) 107.

[28] W. Kohl, B. Witte, G. Ho“fle, Planta Med. 47 (1983) 177.

[29] A. Rehwald, O. Sticher, Phytochem. Anal.6 (1995) 96.

[30] M.J. Donaldson, H. Broby, M.W. Adlard, C. Bucke, Phyto-chem. Anal. 1 (1990) 18.

[31] B. Dra"ger, Phytochem. Anal.6 (1995) 31.

[32] N. Tanaka, K. Kimata, K. Hosoya, H. Miyanishi, T. Araki, J. Chromatogr. A 656 (1993) 265.

[33] L.A. Ciolino, J.A. Turner, H.A. McCauley, A.W. Smallwood, T.Y. Yi, J. Chromatogr. A 852 (1999) 451

[34] M. Unger, J. Chromatogr.A 807 (1998) 81.

[35] Shrivastava N, Padhya MA. Punarnavine profile in the regenerated roots of Boerhaaviadiffusa from leaf segments.CurrSci 1995; 68: 653-656.

[36] Hu CY, Wang PJ. Meristem, shoot-tip and bud culture. In: Evans DA, Wang WR, Ammirato PV, Yamada Y (eds). Handbook of Plant CellCulture, vol 1. New York: MacMillan, 1983, pp 177-277.

[37] Benjamin BD, Roja P, Heble MR, Chadha MS. Multiple shoot cultures of Atropabelladona: effect of physicochemical factors on growth and alkaloid formation. J Plant Nutr.1987; 129: 129-35.

[38] Lal N, Ahuja PS. Plantlet regeneration from callus in PicrorhizakurroaRoyleexBenth. - An endangered medicinal plant. Plant Tissue Cult.1996; 6: 127-34.

[39] Borthakur M, Singh RS. Direct plantlet regeneration from male inflorescences of medicinal yam (Dioscorea floribunda Mart. \& Gal.). In-vitro Cell DevBiol-Plant. 2002; 38: 183-5.

[40] Rai VR. Rapid clonal propagation of Nothapodytesfoetida (wight) sleumer - a threatened medicinal tree. In-vitro Cell DevBiolPlant. 2002; 38: 347-51.

[41] Rout GR, Das P. In-vitroorganogenesis in ginger (ZingiberofficinaleRosc.). J Herbs, Spices and Med Plants. 1997; 4: 41-51

[42] Lutterbach R, Stoockigt J. 1992. High-yield formation of arbutin from hydroquinone by cell-suspension cultures of Rauwolfiaserpentina. HelvChimActa, 75: 2009-2011.

[43] Ruyter CM, Mahmood A, Illahi I, Stockigt J. Investigation of the alkaloid content of Rauwolfiaserpentina roots from regenerated plants. Plant Med 1991; 57: 328-330.

[44] Sreeramu BS, Farooqi AA.Cultivation of medicinal and aromatic crops. Hyderabad: Universities Press; 2001 ; pp 210-211.

[45] Coleman WK, Greyson RI. Root regeneration from leaf cuttings of Lycopersiconesculentum Mill: Application of the leaf plastochron index and responses to exogenous Gibberellic acid. J Exp Bot 1976; 27: 1339-1351.

[46] Attfield EM, Evans PK. Developmental pattern of root and shoot organogenesis in cultured leaf explants of Nicotianatabacum. J Exp Bot 1991; 42: 51-57.

[47] Tran Thanh Van M, Chlyah H, Chlyah A. Tissue culture and plant science. London: Academic Press; 1974. pp. 174.

[48] Stockigt J. 1995. Biosynthesis in Rauwolfiaserpentina, modern aspects of an old medicinal plant. Alkaloids, 47: $115-172$.

[49] Falkenhagen H, Polz L, Takayama H, KitajimaM, Sakai S I,Aimi N, Stöckigt J. 1995. Substrate specificity of vinovine hydroxylase.A novel membrane-bound key enzyme or Rauwolfiaindole alkaloids biosynthesis.Heterocycles, 41: 2683-2690.

[50] Lutterbach R, Stockigt J. 1995. Dynamics of the biosynthesis of methyl ursubin in plant cells emplyingin vivo 13C NMR without labelling.Phytochem, 40: 801-806.

[51] Schröder C, Lutterbach R, Stöckigt J. 1996. Preparative biosynthesis of natural glucosides and fluorogenic substances for bglucosidases followed by in vivo 13C NMR with high density plant cell cultures. Tetrahedron, 52: 925-934.

[52] Sajc, L., D. Grubisic, and G. Vunjak-Novakovic. 2000. Bioreactors for plant engineering: an outlook for further research. Biochem. Eng. J. 4: 89-99.

[53] Kieran, P.M., P.F. MacLoughlin, and D.M. Malone. 1997. Plant cell suspension cultures: some engineering considerations J. Biotechnol. 59: 39-52.

[54] Gantet, P. and J. Memelink. 2002. Transcription factors: tools to engineer the production of pharmacologically active plant metabolites. Trends Pharmacol. Sci. 23: 563-569.

[55] Hiatt, A., R. Cafferkey, and K. Boedish. 1989. Production of antibodies in transgenic plants. Nature 342: 76-78.

[56] Rastogi R. P., Mehrotra B. N, Compendium of Indian Medicinal Plants I: 1960-1969: CDRI, Lucknow and Publications and Information Directorate, New Delhi, 1993, p.338-343.

[57] Davey R, McGregor J.A, Grange J.M., Quality control of homoeopathic medicines; (1), British Hom. J; 1: $1992 \mathrm{a}$ : 81, $78-81$.

[58] Edeoga, H.O., D.E. Okwu and B.O. Mbaebie, 2003.Minerals and nutritive values of some Nigerian medical plants. J. Med. Aromatic Plants Sci., 25: 1010-1015 